CORPUS $\begin{aligned} & \text { Corpus } \\ & 21 \mid 2020 \\ & \text { Dispositifs numériques et dévoilement de soi }\end{aligned}$

\title{
Spécificités de communication des forums de discussion
}

Specificities of communication in discussion forums

\section{Erika Lombart et Cédrick Fairon}

\section{(2) OpenEdition}

Journals

Édition électronique

URL : http://journals.openedition.org/corpus/5356

DOI : $10.4000 /$ corpus.5356

ISSN : 1765-3126

Éditeur

Bases; corpus et langage - UMR 6039

Référence électronique

Erika Lombart et Cédrick Fairon, "Spécificités de communication des forums de discussion 》, Corpus [En ligne], 21 | 2020, mis en ligne le 09 mars 2020, consulté le 24 janvier 2021. URL : http:// journals.openedition.org/corpus/5356; DOI : https://doi.org/10.4000/corpus.5356

Ce document a été généré automatiquement le 24 janvier 2021.

(c) Tous droits réservés 


\title{
Spécificités de communication des forums de discussion
}

\author{
Specificities of communication in discussion forums
}

Erika Lombart et Cédrick Fairon

1 Le Web 2.0, aussi connu sous le nom de Web participatif ou de web social (Mangenot et Soubrié, 2015:3), se caractérise par des contenus numériques générés par tout un chacun grâce aux nouvelles technologies et à leur accès facile. L'activité sociale y tient une place centrale (Levin et Bryan, 2008) via la communication médiée par ordinateur définie comme «le processus par lequel les utilisateurs créent, échangent et comprennent l'information via l'utilisation de systèmes de télécommunication en réseau permettant l'encodage, la transmission et le décodage de messages » (CMC, December, 1996, 1997). Au sein de la CMC se trouve la communication écrite médiée par ordinateur (cémo, Panckhurst, 1997). Celle-ci correspond aux messages numériques écrits caractérisés par les limites et les particularités des environnements numériques dans lesquels ils sont produits (Paveau, 2013).

2 C'est précisément sur un environnement numérique particulier que cet article entend se pencher en répondant à la question de recherche suivante: quelles sont les spécificités de communication des forums de discussion en tant qu'environnement numérique de la cémo ? Si les forums ont déjà fait l'objet de nombreuses analyses, celleci présente l'avantage de les replacer au sein d'un tableau de qualification soulignant les aspects pragmatiques et sémantiques spécifiques à chacun des environnements et pouvant servir de base théorique fiable à une analyse comparative de corpus.

\section{Cémo et cadre théorique}

3 Les messages de la cémo peuvent la plupart du temps être qualifiés de messages éphémères et rapidement rédigés au détriment de la correction linguistique (Silva, 2011); bien que cela reste une généralité dans laquelle il est difficile de faire entrer certains environnements comme les Wiki où le niveau de langue est souvent très élevé. 
On entend ici par environnement, environnement numérique, c'est-à-dire « un espace structuré par des instruments technologiques divers, permettant aux usagers d'accéder à des ressources et à des services numériques présents sur les machines ou en ligne " (INDECU, 2018). Au sein de ces espaces et de leur structure particulière se développent ce que Paveau (2013) nomme les " technogenres ", ou genres numériques, que l'on peut classer en 3 types: (1) les genres prescrits qui désignent les discours produits dans un environnement fortement contraint par la technologie comme twitter et son nombre de caractères limité, (2) les genres négociés qui qualifient les genres qui existaient déjà dans l'univers prénumérique, mais qui ont trouvé leur place dans l'univers du Web 2.0 comme les listes du pire ou du meilleur que l'on trouve en abondance sur Facebook et dans lesquelles le site Topito ${ }^{1}$ 'est spécialisé, et finalement (3) les genres produsés qui sont des genres natifs d'internet, non soumis aux contraintes des technogenres prescrits et nés des nouvelles possibilités technologiques.

Bien que la liste qui suit a de grandes chances de devenir rapidement obsolète vu le rythme des inventions technologiques, nous distinguerons ici 9 environnements de production des messages de la cémo que sont les blogs, les forums de discussion, les réseaux sociaux, les (espaces de) commentaires, les sms, les messageries instantanées, les chats, les courriers électroniques et les wikis (sites web librement modifiables par des utilisateurs ne disposant d'aucune compétence technique particulière [Delacroix, 2005]).

5 Ces nouveaux environnements numériques représentent à la fois une opportunité jamais rencontrée jusqu'à présent et un véritable défi (Poudat et al., 2020). D’une part, jamais les chercheurs n'ont eu accès à des corpus présentant une telle quantité de données variées, tant au niveau des éléments linguistiques et extralinguistiques, que des métadonnées et des historiques de conversations accessibles. D'autre part, l'analyse conversationnelle et pragmatique de tels corpus doit s'inscrire dans une approche nouvelle prenant en compte l'hybridité des discours et des genres encore instables, et repensant la structure d'une interaction impliquant des locuteurs multiples. L'analyse de corpus issus des environnements numériques doit pouvoir s'appuyer sur une méthodologie adaptée, résultant d'une véritable réflexion permettant une approche scientifique de ces nouvelles réalités. C'est à cela qu'a œuvré Thierry Charnier, qui s'est beaucoup impliqué dans la création d'un corpus à l'image des différents aspects de la cémo $(\mathrm{CoMeRe})$ et à qui le précédent numéro de Corpus (Corpus 20, 2020) rend hommage.

Les corpus issus des forums présentent eux aussi des caractéristiques et des défis particuliers. Les membres d'un forum participent à l'écriture collective d'un document (Lewkowicz \& Zacklad, 1999). Le forum peut en effet être défini comme un document numérique conversationnel dans la mesure où il rassemble des informations relatives à une thématique sous la forme d'une conversation (Marcoccia, 2001). L'analyse de cet environnement hybride doit prendre en compte 4 spécificités liées à la caractérisation du corpus, au type d'interaction, à la structuration des échanges et au cadre participatif (Marcoccia, 2004). (1) La caractérisation du corpus. Les forums constituant en quelque sorte une conversation sans début ni fin, la question de la délimitation du corpus se pose. Par ailleurs, le corpus peut avoir subi l'intervention d'une modération qui a pu effacer des messages dont elle a laissé les réponses qui deviennent difficiles, voire impossibles, à analyser. Les échanges peuvent également présenter des traces d'interdiscours (Todorov, 1981) établissant des relations entre le discours analysé et 
d'autres discours, et d'histoires conversationnelles (Golopenja-Erescu, 1988) nées de précédents échanges et influençant les échanges en cours entre individus. (2) Le type d'interaction. L'interaction est sans fin et ses participants évoluent au fil des messages. Elle se présente sous la forme de discussions souvent désorganisées avec un temps de latence parfois très important entre deux messages. (3) La structuration des échanges. Les messages n'ont pas tous le même statut. Certains sont initiatifs dans la mesure où ils instaurent parfois l'échange (bien que de nombreux messages initiatifs restent sans réponse) tandis que d'autres, réactifs, y répondent et peuvent parfois devenir euxmêmes initiatifs d'une nouvelle discussion. (4) Le cadre participatif. Les participants aux échanges ne sont pas non plus sur un pied d'égalité que ce soit en production ou en réception. Certains d'entre eux produisent des messages, tandis que d'autres, les lurkers, se contentent de les lire. Le statut des destinataires est lui aussi hétérogène puisqu'un message peut s'adresser à un ou plusieurs destinataires directs, tout en ayant également des destinataires secondaires dits privilégiés et de simples témoins.

\section{Environnements de la cémo et critères de qualification}

Chaque environnement a son propre genre de communication avec des règles explicites et implicites (Poudat et Ho-Dac, 2019 ; Cougnon et Bouraoui, 2017). Bien que certains chercheurs tiennent également compte de caractéristiques telles que la limitation des caractères ou la visibilité (Verheijen, 2018), les 4 critères généralement retenus dans les tentatives de catégorisation des environnements de la cémo sont le support, le but, les acteurs et la synchronicité (Cougnon, 2015, Marcoccia, 2005). La suite de cet article sera consacrée à leur présentation, leur discussion, leur actualisation et leur complétion.

\section{Critère 1 : support}

8 Il y a quelques années, il était pertinent de classer les différents environnements de la cémo en fonction de leur support et de distinguer les messages échangés par ordinateurs de ceux composés sur les téléphones portables. Mais les évolutions technologiques ont rendu ce critère d'analyse moins pertinent. On peut ainsi faire aujourd'hui sur un smartphone à peu près tout ce qu'on peut faire sur un ordinateur et l'apparition des tablettes est venue brouiller davantage les frontières entre les différents supports. Ce phénomène va de pair avec la « convergence numérique » qui a pour conséquence, comme le souligne Fairon (2014), de regrouper sur un même support toutes les formes de communication. Ce phénomène, aussi appelé "convergence médiatique " (Caron et Caronia, 2005) vient remettre en question les spécificités communicationnelles liées aux différents supports qui restent présentes - on pense notamment à la facilité de production d'un message liée à la taille des claviers - mais tendent à disparaitre et rendent la question du support beaucoup plus complexe ; raison pour laquelle nous ne retiendrons pas ce premier critère.

\section{Critère 2 : but de la communication}

9 Jakobson (1960) a défini les 6 fonctions de la communication que sont les fonctions expressive, conative, phatique, métalinguistique, référentielle et poétique. Chacune d'elles met en avant l'une des composantes de l'échange, à savoir le destinateur 
(fonction expressive), le contexte (fonction référentielle), le message (fonction poétique), le contact (fonction phatique), le code (fonction méta-linguistique) et le destinataire (fonction conative).

Les messages produits dans le cadre de la cémo peuvent eux aussi se situer au sein de cette classification. Ainsi ces messages peuvent servir à communiquer une émotion ou une attitude, transmettre une information ou se référer au contexte (qui sera bien sûr particulier puisqu'il n'est pas physiquement présent), jouer avec la langue, établir un contact avec l'autre, utiliser la langue pour parler de la langue ou encore tenter de faire (ré)agir le destinataire. Les différents environnements sont flexibles et peuvent accueillir des contenus avec des objectifs différents. Cette cohabitation relève de la convergence numérique, cette fois définie par Jenkins (Jenkins 2006 dans Kerneis et al., 2012) comme la connexion toujours plus présente de contenus médiatiques différents pris en compte dans la construction du sens. Notons que, si les environnements ne sont pas figés, certains d'entre eux se prêtent à la production de certains contenus. David Crystal faisait déjà cette observation en 2001 tandis qu'il définissait le chat comme un médian plus adapté aux échanges d'informations à valeur sociale; observation que Louise-Amélie Cougnon fera également en 2005 au sujet des services de réseautage lorsqu'ils ne sont pas professionnels. L'importance des différentes fonctions de la communication varie donc d'un environnement à l'autre.

11 Les forums de discussion ont longtemps été considérés comme des environnements accueillant des échanges essentiellement orientés vers des tâches professionnelles ou d'apprentissage (Panckhurst 2007). À ce titre, ils s'inscriraient surtout dans la fonction référentielle et présenteraient un faible contenu relationnel (Crystal 2001). Si cette assertion a pu être vraie, l'évolution des pratiques l'a rendue obsolète. En effet, les forums de discussion se caractérisent entre autres par leur communauté d'utilisateurs. Or, la construction de telles communautés passe d'office par une forme d'intimité nécessaire au dévoilement de soi, et donc par le partage d'émotions et d'avis s'inscrivant dans d'autres fonctions de la communication comme le montrent les exemples ci-dessous :

\section{Exemple 1}

vous etes en train de dire que parce que l'alcool et le tabac font plus de ravages qu'il

faut pas l'interdire? mais apres; le cannabis en fera autant de ravages et ce sera de votre faute! Autant prévenir que guérir non?

[Psychologie_Ados_débat pour ou contre la légalisation du cannabis ms surtout pourquoi_ineedhelp]

Exemple 2

... ce soir après avoir chercher toute la journée, elle a reçue une fessée je suis dégoutée ce n'est pas l'éducation que je veux lui transmettre la violenceest une attitude fascisante qui bloque l'enfant encore plus car incapacite de comprendre ces émotions [Psychologie_parents_help ma fille de 3 ans me pousse a bout_profil supprimé]

12 L'exemple 1 présente l'avis d'un utilisateur sur la consommation du cannabis et s'inscrit dans la fonction conative puisqu'il vise à convaincre le récepteur tandis que le message de l'exemple 2 traite des difficultés d'une maman avec sa fille et s'inscrit dès lors dans la fonction expressive. 


\section{Critère 3 : acteurs}

13 Tandis que certains messages de la cémo participent à une communication de masse et ne s'adressent donc à personne en particulier, d'autres mettent par défaut deux participants en relation, tandis que d'autres encore impliquent souvent plus de deux participants (Marcoccia, 2005). En quoi le nombre d'acteurs impliqué dans l'échange vat-il influencer le contexte de communication des différents environnements ? C'est ici qu'interviennent les notions d'intimité et d'extimité.

14 L'intimité qualifie un type particulier de relation qui relève de la mise en place et de la construction d'un lien intime, privilégié (Chavalérias, 2003). Elle est donc ce que nous rendons accessible à quelques-uns et il n'est pas concevable de parler d'intimité dans le cadre d'une communication de masse. L'intimité se retrouve davantage dans les environnements permettant une relation interpersonnelle impliquant par défaut 2 participants et plus. "L'extimité » est un terme provenant du domaine de la psychanalyse où elle est définie comme « le mouvement qui pousse chacun à mettre en avant une partie de sa vie intime pour mieux se l'approprier en l'intériorisant sur un autre mode grâce aux échanges qu'elle suscite avec les proches » (Tisseron, 2011 : 52). Ce processus repose sur trois étapes que sont l'extériorisation d'éléments intimes, la validation de ce vécu par autrui, et le bénéfice personnel et social qui en est tiré (Paveau, 2017). L'extimité est ainsi au cœur du dévoilement de soi, étape nécessaire à la création d'une vie sociale et d'une communication riches. Si le dévoilement de soi n'est pas inenvisageable dans une communication de masse, il trouve dans les environnements propices aux relations interpersonnelles impliquant par défaut 2 participants et plus un contexte de communication facilitant son accomplissement. Il est à noter qu'en ligne, il est difficile de maintenir une séparation nette entre ce qui est privé et public étant donné que les sites sont généralement constitués d'espaces mixtes (Côté, 2012).

15 Les forums de discussion peuvent être définis comme des dispositifs hybrides de communication interpersonnelle de masse (Baym 1998) dans la mesure où ils permettent à la fois l'échange interpersonnel et la communication de masse. Le premier message d'un fil de discussion, posté tel une bouteille à la mer, appartient à la communication de masse. L'émetteur s'adresse aux nombreux autres utilisateurs partageant un centre d'intérêt ou ses préoccupations, mais à personne en particulier. Les réponses qui seront apportées à ce premier message relèvent quant à elles de la relation interpersonnelle impliquant par défaut 2 participants et plus.

Doit-on parler d'intimité ou d'extimité dans le cadre des forums? On parlera clairement d'extimité, et cela d'autant plus dans le cadre des forums de santé et des spécificités liées au soutien social. Pourtant la question n'est pas si simple comme le montre l'illustration ci-dessous ${ }^{2}$ (Figure 1) tirée d'une présentation interne à la société Doctissimo qui s'inquiétait de l'éventuelle obsolescence des forums face aux nouveaux environnements du web, comme les réseaux sociaux.

Figure 1. Capture d'écran de la page 9 du Power Point présenté aux modérateurs de Doctissimo en 2016. (Document non publié) 


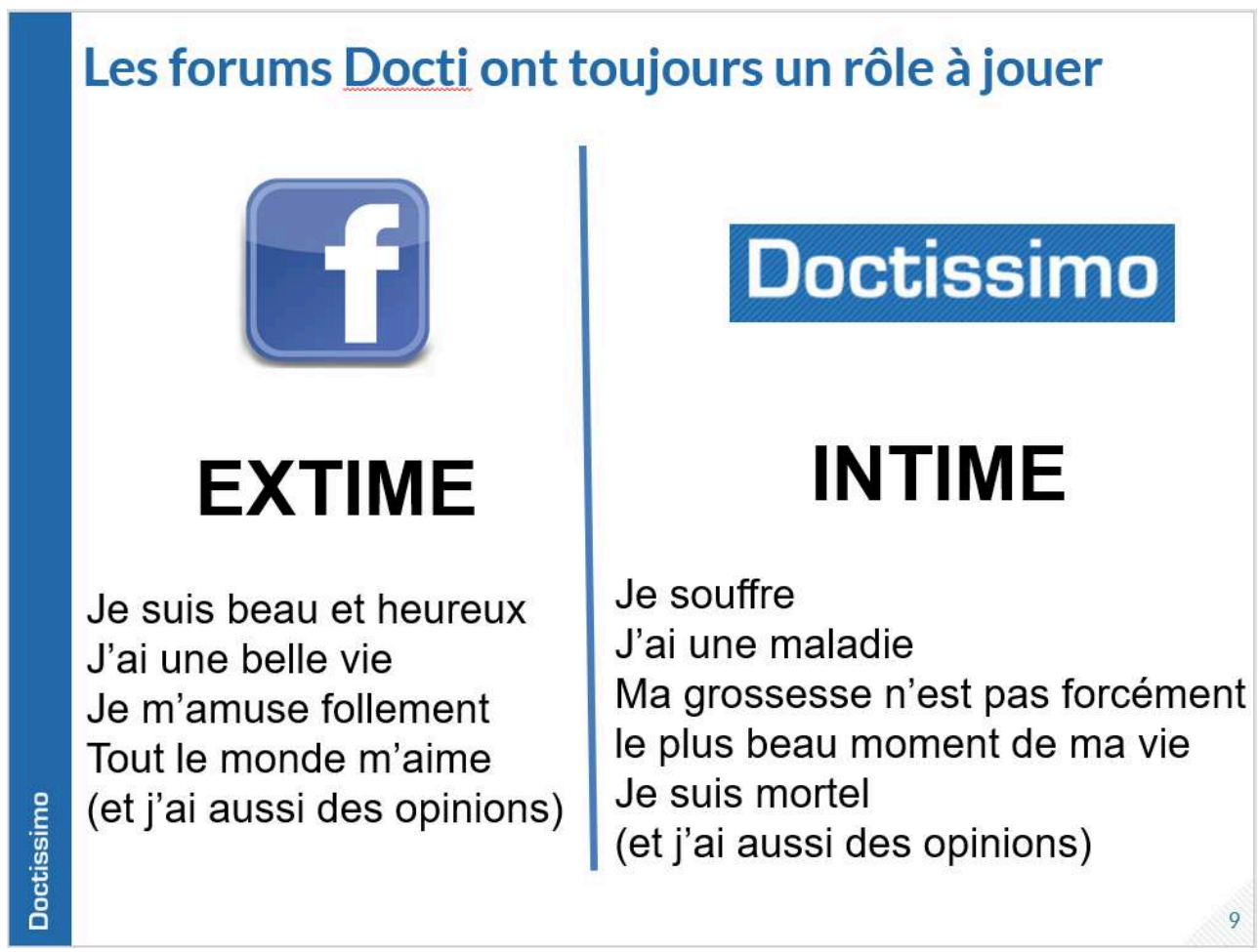

Selon Doctissimo, les forums dans lesquels les participants partagent des aspects négatifs de leur vécu comme la peur et la maladie relèvent de l'intimité à l'opposé de Facebook où les participants partagent/mettent en scène des aspects positifs de leur vie et qui se caractériserait par son caractère extime. En réalité, dans les deux cas, il s'agit d'extimité, mais avec des objectifs différents. Tandis que le partage d'émotions et d'expériences négatives sur les forums permet d'obtenir un soutien social, la mise en scène des aspects positifs de sa vie permet à l'utilisateur de Facebook de se construire une identité numérique parfois assez éloignée de son identité réelle. En résumé, si l'extimité permet à l'utilisateur des forums d'obtenir du soutien, elle permet aussi à celui de Facebook (et plus largement, des réseaux sociaux) de provoquer l'admiration.

\section{Critère 4 : (a)synchronie}

Les messages de la cémo peuvent être quasi-synchrones ou asynchrones. Ils offrent donc la possibilité d'une discussion en temps réel où les interlocuteurs échangent de manière instantanée, mais permettent également une réponse différée. Avec Garcia et Jacobs (1999), nous privilégierons le terme de quasi-synchronicité à celui de synchronicité dans la mesure où - dans les limites des applications actuelles - le/les destinataires ne peut/peuvent avoir accès au message produit qu'une fois celui-ci posté et non de manière simultanée à sa production comme c'est le cas d'un message vocal.

Si cette dichotomie synchrone/quasi-synchrone est pertinente, elle ne suffit pas pour représenter la complexité des échanges de la cémo qui prennent en compte des facteurs relationnels ou contextuels. Ainsi, le temps de latence acceptable d'une réponse varie fortement en fonction de la relation (personnelle ou professionnelle) que le destinataire entretient avec l'émetteur et du caractère contextuel ou urgent du contenu de l'échange. Un sms ayant pour message «Est-ce que je peux passer dans 5 minutes? » demande par défaut une réponse endéans les 5 minutes. Le caractère 
asynchrone ou quasi-synchrone de l'échange doit être envisagé sous forme d'un continuum temporel dans lequel le temps de réponse acceptable à un message se rapproche ou non de zéro. Un environnement sera donc perçu comme généralement asynchrone ou généralement quasi-synchrone, et cette caractérisation n'est pas sans conséquence.

20 (1) Échanges quasi-synchrones. Au niveau linguistique, les messages sont rapidement rédigés dans un style informel et l'attention accordée à l'orthographe, à la ponctuation et à la grammaire est moindre (Baron et Ling, 2007). Au niveau relationnel et social, la quasi-synchronicité accroît la spontanéité des réponses et des thématiques (Cougnon, 2015). Mais cette spontanéité rend les échanges plus compliqués à analyser car ils demandent souvent un démêlage (Crystal, 2001) lié aux tours de paroles qui peuvent être complexes à cause de l'imbrication des messages et de la relecture a posteriori.

(2) Echanges asynchrones. Le temps qui s'écoule entre le moment où un message est posté et celui où il recevra une réponse peut être plus ou moins long. Ce temps de latence a plusieurs conséquences. La première est interactionnelle car un environnement asynchrone laisse à chaque utilisateur le temps dont il a besoin pour répondre et participer sans être exclu de la conversation s'il est plus lent (Andresen, 2009). Selon Wise et al. (2006), cette caractéristique rend les messages asynchrones particulièrement adaptés à la construction de communautés virtuelles dans la mesure où ils autorisent un certain temps de réflexion, ne demandent pas à tous les membres de la communauté d'être présents au même moment, et augmentent la crédibilité et l'engagement de l'auteur qui a cependant l'impression d'être moins utile et persuasif que lors d'un échange synchrone (Wise et al., 2006). La seconde conséquence est linguistique. En diminuant la spontanéité des échanges, l'asynchronicité augmente le temps de réflexion mais aussi celui, éventuel, de la révision sémantique, grammaticale ou orthographique. Les messages présents sur un forum sont généralement plus longs et mieux construits que ceux que l'on peut trouver dans un environnement quasisynchrone.

L'opposition quasi-synchrone et asynchrone doit être complétée par la notion de commentaire. L'environnement dialogique apparait lorsqu'un utilisateur poste un message et que les réponses des autres utilisateurs ont le même statut que lui. Le tout forme alors une discussion comme c'est le cas des sms, des messageries instantanées ou des mails. D'autres environnements fonctionnent sur la base, non pas d'un dialogue, mais de statuts et de commentaires (Cougnon, 2015) qui ne sont pas à mettre sur un même pied. Le dialogue n'apparaît que lorsque les commentaires se répondent entre eux et forment alors un autre environnement de la cémo, celui de l'espace de commentaire, qui tient davantage du forum si les échanges sont asynchrones ou du chat si les utilisateurs se mettent à communiquer en temps réel. Ainsi, non seulement différents environnements, dialogiques ou non, peuvent cohabiter au sein d'un même espace, mais un environnement non dialogique peut également se transformer en environnement dialogique. Le critère de (quasi)synchronicité ne sera étudié que dans le cadre des environnements dialogiques.

23 Les forums de discussion forment un environnement dialogique asynchrone. De cette asynchronie naissent au moins deux caractéristiques spécifiques. (1) les sujets de discussion restant en permanence accessibles, les conversations ne sont jamais définitivement closes (sauf choix éditorial contraire) et les sujets peuvent donner l'impression d'être morcelés (Battaïa, 2016). Goffman (1987) parle à ce sujet de 
« conversations chroniquement en cours ». Les messages des forums présentent par ailleurs un nombre de mots relativement important (Panckhurst, 2006). (2) Il est fréquent que des messages restent sans réponse, tandis que d'autres revêtent un caractère monologique, et donc sans prise en compte de l'interlocuteur (Marcoccia et Gauducheau, 2007). Cet aspect rend l'analyse du processus d'interprétation plus complexe puisque, sans réponse, on ne peut savoir comment le message a été reçu et entreprendre une analyse dialogique puisqu'il n'y a pas d'échange.

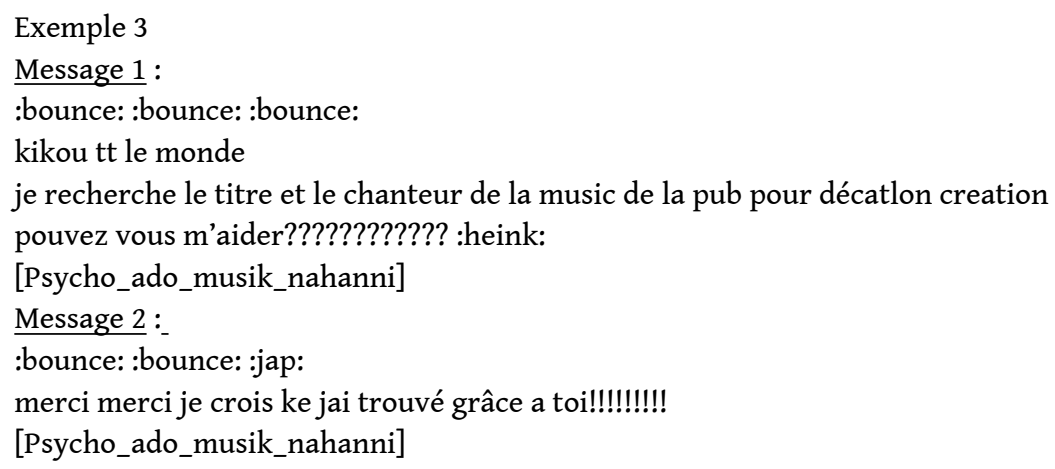

Comme le montre l'exemple 3, certaines discussions restent en attente d'une réponse. Dans ce cas-ci, l'utilisateur s'est répondu à lui-même un jour après avoir posté un message auquel personne n'a prêté attention.

\section{Critère 5 : pseudonymat}

Certains environnements de la cémo sont caractérisés par le pseudonymat, tandis que d'autres impliquent que les participants connaissent leur identité réciproque. L'échange sous pseudonymat peut présenter des caractéristiques particulières liées au sentiment d'une absence de responsabilité. Mais même si l'identité de la personne n'est pas directement accessible, l'anonymat n'existe pas sur internet - sauf sur le dark web puisqu'il est toujours possible de remontrer jusqu'à l'adresse IP de l'utilisateur. On parlera donc plutôt de "pseudonymat " qui consiste en l'adoption d'un pseudonyme qui garantit à l'utilisateur que son identité officielle ne sera pas révélée sans pour autant le dispenser de répondre de son utilisation (Plateau et Lacharme, 2012) et relève de l'identité numérique à laquelle il participe. Les pseudos n'ont pas seulement pour but de masquer l'identité de l'émetteur, mais aussi d'enrichir son identité numérique. Ainsi, si le choix du pseudo est soumis à des contraintes techniques (deux pseudos identiques ne peuvent par exemple pas cohabiter sur un même site [Anis, 2001]), il est loin d'être anodin puisqu'il est issu d'un processus porteur de sens visant à la désignation de soi comme sujet social (Emerit, 2014). Le pseudo permet donc de masquer l'identité civile tout en étant un lieu d'identification (Perea, 2010 ; Barratt, 2012). Gauducheau (2012) et Walther (1996) soulignent que le pseudonyme peut être une source d'information sur l'identité des utilisateurs, tout comme leur manière de s'exprimer, et que les personnes ayant noué des relations intimes sur les forums échangent également via d'autres moyens de communication plus intimes, voire en face-à-face. Le pseudonymat présente des spécificités énonciatives et discursives. L'idée du dédoublement de l'instance de parole et de dualisme numérique, déjà présente chez Turkle (1984), est reprise par Doueihi (2008) et traite du fait que le passage du connecté au non connecté modifie les caractéristiques de l'énonciation qui reste régulée pour mener la communication à bien. 
L'impact du pseudonymat sur la communication est double.

(1) Impact négatif. L'anonymat, et avec lui le pseudonymat, intensifie la cyberviolence verbale (Paveau, 2017), favorise le dévoilement d'expériences émotionnelles négatives grâce à la liberté que les participants se sentent autorisés à prendre par rapport aux jugements potentiels (Walther et Boyd, 2002), encourage le mensonge et la dissimulation, rend plus compliqué l'établissement d'une relation de confiance (Tanis et Postmes, 2005) et déshumanise l'échange (Pan et al., 2018; Taylor, 2010). On peut ainsi remarquer une fréquence plus élevée de commentaires en ligne injurieux ou hostiles (Kiesler et al., 1984 ; Johnson, 2010) comme l'a fait Santana (2014) dans une étude selon laquelle 53,3\% des commentaires produits anonymement présentent des propos vulgaires, racistes, haineux ou blasphématoires contre seulement $28,7 \%$ des commentaires non-anonymes. Ce phénomène se nomme "effet de désinhibition en ligne ». L'anonymat influence ainsi le comportement du locuteur qui n'assume pas toujours sa responsabilité énonciative et les conséquences de son discours sur un interlocuteur physiquement absent (Temmar, 2012). Catherine Blaya (2013), qui se penche davantage sur le comportement des plus jeunes, parle quant à elle «d'effet cockpit » pour décrire cette situation d'énonciation particulière dans laquelle, tel un aviateur, le locuteur lance une bombe sur une cible qu'il n'identifie que vaguement.

(2) Impact positif. L'identité d'emprunt présente cet avantage d'augmenter l'impression de sécurité ressentie par les utilisateurs (Pan et al., 2018) et de leur permettre de se cacher tout en s'autorisant à s'investir davantage (Colas-Blaise et Halté, 2011). Ainsi, l'anonymat/pseudonymat permet à des individus, qui ne l'avaient pas dans le monde prénumérique d'avoir accès à la parole (Johnson, 2010), et de pouvoir faire vivre un moi numérique au sein de communautés à travers la création et le maintien de liens affectifs (Paveau, 2017). C'est ce que Suler (2004) appelle l'effet de désinhibition de l'anonymat qui a donc un impact positif en participant au dévoilement de soi nécessaire à la construction des relations sociales (Berger, 2002 cité dans Gauducheau, 2012). Une plus grande conscience de soi induite par l'anonymat mène ainsi les utilisateurs des forums à faire davantage part de leurs ressentis et de leurs expériences personnelles (Joinson, 2001 cité dans Gauducheau, 2012). L'anonymat favorise l'intimité (Maltby et al., 2018).

Dans les forums de discussion, les utilisateurs communiquent généralement sous pseudonymat. Comme le montrent les exemples ci-dessous, les phénomènes (négatifs) de cyberviolence verbale et (positif) de désinhibition s'y retrouvent.

(1) Le flaming est un comportement agressif poussé à l'extrême qui se définit par le fait de provoquer et d'insulter les autres usagers sans raison apparente (Herring et al., 2002). Il s'accompagne généralement de nombreuses insultes et émoticônes négatives.

\section{Exemple 4}

Espèce de salope,si je vois ta gueule,je te la défonce. Crève sale merde et oui,t'es bel et bien une pourriture qui ne vaut rien.

[Psychologie_Coup de gueule_marre de l'agressivité des gens_Profil Supprimé]

\section{(2) La désinhibition}

\section{Exemple 5}

Nous arrivons à en parler ensemble, tous, les plus jeunes avait 8 et 10 ans quand leur grand frère est mort, mais ils en parlent très facilement, sans tabou. C'est parfait. Pour eux. Mais, et c'est pour cela que je suis de retour sur docti, c'est très dur de ne pas fondre en larmes devant eux quand ils prononcent son prénom, c'est impossible de leur dire que même si je les aime autant que j'aimais et j'aime Olivier, 
une grosse partie de mon coeur est morte il y a deux ans. Et que ma vie ne m'importe plus. Je ne reste auprès d'exu que parce que je sais qu'ils auraient trop de mal à revivre cet "abandon".

Alors, excusez moi encore de venir m'épancher ici, mais l'anonymat libère.

[Psychologie_Perdre un enfant _ deux ans..._lis29oy]

\section{Tableau de qualification et perspectives}

L'analyse que nous avons opérée ci-dessous est générale et représente les tendances majoritairement présentes dans les différents environnements. Cette généralisation n'enlève rien au fait qu'une personne, à cause de son caractère, de sa situation professionnelle ou d'une période particulière de sa vie puisse faire un usage particulier de ces environnements. Ainsi, certaines personnes connectées en permanence peuvent répondre directement à leurs mails, transformant un échange habituellement asynchrone en un échange quasi-synchrone, et certaines personnalités ont tellement de followers sur Facebook que leur mur tient plus de la communication de masse.

Figure 2. Tableau synthétique des spécificités de communication des environnements de la cémo

\begin{tabular}{|c|c|c|c|c|}
\hline Environnement & $\begin{array}{l}\text { Fonction de la } \\
\text { communication }\end{array}$ & $\begin{array}{l}\text { Nombre de } \\
\text { participants }\end{array}$ & $\begin{array}{l}\text { Commentaire/ } \\
\text { Dialogue quasi- } \\
\text { synchrone/Dialogue } \\
\text { asynchrone }\end{array}$ & Pseudo \\
\hline Blog public & $\begin{array}{l}\text { Référentielle / } \\
\text { Expressive / } \\
\text { Conative }\end{array}$ & $\begin{array}{l}\text { Communication de } \\
\text { masse } \\
\text { - Extimité }\end{array}$ & Commentaire & Parfois \\
\hline $\begin{array}{l}\text { Forum de } \\
\text { discussion public }\end{array}$ & $\begin{array}{l}\text { Expressive / } \\
\text { Conative / } \\
\text { Référentielle / } \\
\text { Phatique }\end{array}$ & $\begin{array}{l}\text { Communication de } \\
\text { masse/+ de } 2 \\
\text { participants } \\
\text { - Extimité }\end{array}$ & Dialogue asynchrone & Généralement \\
\hline $\begin{array}{l}\text { Réseau social } \\
\text { (privé } \quad \& \\
\text { professionnel) }\end{array}$ & $\begin{array}{l}\text { Référentielle / } \\
\text { Expressive / } \\
\text { Conative }\end{array}$ & $\begin{array}{l}+ \text { de } 2 \text { participants } \\
\text { - Extimité }\end{array}$ & Commentaire & Rarement \\
\hline Commentaire & $\begin{array}{l}\text { Référentielle / } \\
\text { Expressive / } \\
\text { Conative }\end{array}$ & $\begin{array}{l}\text { Communication de } \\
\text { masse/+ de } 2 \\
\text { participants } \\
\text { - Extimité }\end{array}$ & Dialogue asynchrone & Parfois \\
\hline SMS & $\begin{array}{l}\text { Expressive / } \\
\text { Conative / } \\
\text { Référentielle / } \\
\text { Phatique }\end{array}$ & $\begin{array}{l}2 \text { participants et + } \\
\text { - Intimité }\end{array}$ & Dialogue asynchrone & Jamais \\
\hline $\begin{array}{l}\text { Messagerie } \\
\text { instantanée }\end{array}$ & $\begin{array}{l}\text { Expressive / } \\
\text { Conative / } \\
\text { Référentielle / } \\
\text { Phatique }\end{array}$ & $\begin{array}{l}2 \text { participants et + } \\
\text { - Intimité }\end{array}$ & $\begin{array}{l}\text { Dialogue quasi- } \\
\text { synchrone }\end{array}$ & Parfois \\
\hline
\end{tabular}




\begin{tabular}{|l|l|l|l|l|}
\hline Chat & $\begin{array}{l}\text { Expressive / } \\
\text { Conative / } \\
\text { Référentielle / } \\
\text { Phatique }\end{array}$ & $\begin{array}{l}\text { +de 2 participants - } \\
\text { Extimité }\end{array}$ & $\begin{array}{l}\text { Dialogue quasi- } \\
\text { synchrone }\end{array}$ & Généralement \\
\hline $\begin{array}{l}\text { Courrier } \\
\text { électronique }\end{array}$ & $\begin{array}{l}\text { Référentielle / } \\
\text { Expressive / } \\
\text { Conative }\end{array}$ & $\begin{array}{l}\text { 2 participants et + } \\
\text { - Intimité }\end{array}$ & Dialogue asynchrone & Parfois \\
\hline Wiki & Référentielle & $\begin{array}{l}\text { Communication de } \\
\text { masse }\end{array}$ & Commentaire & Parfois \\
\hline
\end{tabular}

Une fois replacées au sein de ce tableau, voici les caractéristiques qui peuvent être attribuées aux forums de discussion:

Les messages postés ont pour but d'échanger des informations et des avis, et de créer des liens sociaux comme le soulignent Paganelli et Clavier (2011). Ils s'inscrivent dans les 4 fonctions de la communication de Jacobson: expressive, conative, phatique et référentielle. Les forums sont à la fois le lieu d'une communication de masse et d'une communication interpersonnelle impliquant par défaut plus de deux participants. Cette communication interpersonnelle, qui apparaît dès le second message posté, favorise l'expression d'une extimité négative, et ce d'autant plus dans les forums de santé où les utilisateurs sont en recherche de soutien social. Les échanges au sein des forums se font de manière asynchrone, ce qui se révèle plus propice à la rédaction de messages plus longs, mieux construits, avec une plus grande attention accordée à la langue et une plus grande légitimité même si cela n'est pas ressenti de cette manière par son auteur. Les utilisateurs des forums s'expriment généralement sous pseudonymat, et sont donc plus susceptibles d'être agressifs ou de partager des expériences intimes, ce que le sentiment de sécurité lié au pseudonymat encourage.

\section{Perspectives}

Le Web 2.0 est constitué de différents environnements qui lui confèrent une grande hétérogénéité devant absolument être prise en compte pour mener une analyse, entre autres conversationnelle et pragmatique, pertinente. Ainsi, les contraintes liées à chaque environnement spécifient les caractéristiques mises en évidence dans ce tableau; et bien que non excluants, les critères que sont le but de la communication, ses acteurs, son asynchronie et le pseudonymat permettent d'établir une base théorique susceptible d'apporter une explication pertinente aux phénomènes conversationnels observés dans le Web 2.0 et de jeter les bases d'une analyse contrastive des différents environnements de la cémo. 


\section{BIBLIOGRAPHIE}

Andresen M. A. (2009). « Asynchronous Discussion Forums: Success Factors, Outcomes, Assessments, and Limitations », Educational Technology \& Society $12: 249-257$.

Anis J. (2001). « Approche sémiolinguistique des représentations de l'ego dans la Communication Médiée par Ordinateur », Langages 35, $144:$ 20-38.

Barratt M. J. (2011). « Discussing Illicit Drugs in Public Internet Forums: Visibility, Stigma, and Pseudonymity ", Proceedings of the 5th International Conference on Communities and Technologies, 159-168.

Battaïa C. (2016). «Information médicale et émotion dans les forums de santé », Les Cahiers du numérique $12,1: 51-72$.

Baym N. K. (1998). « The Emergence of On-Line Community », Cybersociety 2.0: Revisiting ComputerMediated Communication and Community, 35-68.

Beaudouin V. (2002). « De la publication à la conversation », Réseaux 116, 6 : 199-225.

Blaya C. (2013). Les ados dans le cyberespace- prises de risque et cyberviolence. Louvain-la-Neuve : De Boeck.

Caron A. H. et Caronia L. (2005). Culture mobile : Les nouvelles pratiques de communication. Toulouse : PUM.

Chevalérias M.-P. (2003). « Intimité et lien intime », Le Divan familial, 11, 2 : 11-23.

Colas-Blaise M. et Halté P. (2011). « Le geste énonciatif dans le chat. L'interjection, l'acronyme et l'émoticône, ou comment (se) montrer à l'écrit », Le discours et la Langue, 2.2.

Cougnon L.-A. (2015). Langage et sms : Une étude internationale des pratiques actuelles. Louvain-laNeuve : Presses universitaires de Louvain.

Cougnon L.-A. et Bouraoui M. J.-L. (2017). « Orality and Literacy of Telephony and SMS », in K. Bedijs et Ch. Maaß (éd.) Manual of Romance Languages in the Media. Berlin : De Gruyter Mouton : 154-175.

Côté J. (2012). « Les enjeux éthiques de l'utilisation d'internet en recherche : Principales questions et pistes de solutions ", Éthique publique. Revue internationale d'éthique sociétale et gouvernementale 14,2 .

Crystal D. (2001). Language and the Internet. Cambridge : Cambridge University Press.

December J. (1997). « Notes on Defining of Computer-Mediated Communication », CMC Magazine.

December J. (1996). « What is Computer-mediated Communication? », online: https://

www.december.com/john/study/cmc/what.html

Delacroix J. (2005). Les Wikis. Espaces de l'intelligence collective. Paris : M2 Editions.

Doueihi M. (2008). La Grande Conversion numérique. Paris : Le Seuil.

Emerit L. (2014). « Vers une typologie des pseudonymes sur Facebook », Actes du colloque IMPEC $2014: 93-104$

Fairon C. (2014). «SMS, langage et convergence numérique », Pop philosophie 2014. 
Gaglio G. (2010). « Consommation d'informations sur Internet et modulation de la relation aux médecins. Le cas d'aidantes de malades atteints d'une pathologie lourde », Sociologies pratiques 20 : 63-74.

Garcia A. C. et Jacobs J. B. (1999). « The Eyes of the Beholder: Understanding the Turn-Taking System in Quasi-Synchronous Computer-Mediated Communication ", Research on Language and Social Interaction 32, 4 : 337-367.

Gauducheau N. (2012). « La communication des émotions dans les échanges médiatisés par ordinateur : Bilan et perspectives », Bulletin de psychologie 496, 4 : 389-404.

Goffman E. (1987). Façons de parler. Paris : Editions de Minuit.

Golopentia-Erescu S. (1988). « Interaction et histoire conversationnelle », in J. Cosnier, N. Gelas et C. Kerbrat-Orecchioni, Échanges sur la conversation. Paris : Editions du CNRS.

Herring S., Job-Sluder K., Scheckler R. et Barab S. (2002). « Searching for Safety Online: Managing "Trolling" in a Feminist Forum », The Information Society 18, 5 : 371-384.

INDECU. (2018). « Environnement numérique », EspaceTemps.net [en ligne], https:// www.espacestemps.net/articles/environnement-numerique/

Jakobson R. (1960). Essais de Linguistique générale. Paris : Editions de Minuit.

Johnson M. (2010). « Anonymity in online discussion forums- does it promote connections? », Proceedings of the 7th International Conference on Networked Learning, 198-206.

Kerneis J., Coutant A., Assogba H. et Stenger T. (2012). « Les natifs numériques profitent-ils de la convergence ? Constats nuancés et pistes de réflexion pour les éducateurs »Études de communication 38 : 53-68.

Kiesler S., Siegel J. et McGuire T. W. (1984). « Social psychological aspects of computer-mediated communication », American Psychologist 39, 10 : 1123-1134.

Levin A. et Bryan A. (2008). «Web 2.0 Storytelling: Emergence of a New Genre », Educause Review $43,6: 40-56$.

Lewkowicz M. et Zacklad M. (1999). «L'écriture collective d'une argumentation dans la prise de décision. Un outil de traçabilité des connaissances en conception ", Document numérique, 3, 3-4 : 263-282.

Ling R. et Baron N. (2007). « Text Messaging and IM Linguistic Comparison of American College Data », Journal of Language and Social Psychology $26: 291-298$.

Mangenot F. et Soubrié T. (2014). « Le web social au service de tâches d'écriture », Recherches 60 : 89-105.

Marcoccia M. (2001). «L'animation d'un espace numérique de discussion : L'exemple des forums usenet », Document numérique, 5,3:11-26.

Marcoccia M. (2005). « La communication médiatisée par ordinateur. Problèmes de genres et de typologie ", Actes de la Journée d'études Les genres de l'oral, ICAR.

Marcoccia N. et Gauducheau N. (2007). «L'analyse du rôle des smileys en production et en réception : Un retour sur la question de l'oralité des écrits numériques ", Glottopol, revue de sociolinguistique en ligne $10: 39-55$.

Pan W., Feng B. et Skye Wingate V. (2018). « What You Say Is What You Get: How Self-Disclosure in Support Seeking Affects Language Use in Support Provision in Online Support Forums », Journal of Language and Social Psychology 37,1: 3-27. 
Panckhurst R. (1997). « La communication médiatisée par ordinateur ou la communication médiée par ordinateur? » Terminologies nouvelles $17: 56-58$.

Panckhurst R. (2006). « Le discours électronique médié : Bilan et perspectives ", Psychologie. Lire, Écrire, Communiquer et Apprendre avec Internet, 345-366.

Panckhurst R. (2007). « Discours électronique médié : Quelle évolution depuis une décennie ? ", in G. Jeannine (éd.) La langue du cyberespace : De la diversité aux normes. Paris : L'Harmattan, 121-136.

Paveau M.-A. (2013). « Genre de discours et technologie discursive. Tweet, twittécriture et twittérature », Pratiques, Théories et pratiques des genres 157-158 : 7-30.

Paveau M.-A. (2017). L'analyse du discours numérique : Dictionnaire des formes et des pratiques. Paris : Hermann.

Perea F. (2010). «L'identité numérique : De la cité à l'écran. Quelques aspects de la représentation de soi dans l'espace numérique », Les Enjeux de l'information et de la communication 1 : 144-159.

Plateaux A. et Lacharme P. (2012). « Organisation d'une architecture de santé respectueuse de la vie privée », $7^{e}$ Conférence sur la Sécurité des Architectures Réseaux et Systèmes d'Information (SAR SSI).

Poudat C. et Ho-Dac L.-M. (s. d.). « Désaccords et conflits dans le Wikipédia francophone », COgniton, REprésentation, LAngage 31, à paraître.

Poudat C., Wigham C. R. et Liégeois L. (2020). «Les corpus de la communication médiée par les réseaux : Une introduction », Corpus, 20. En ligne : http://journals.openedition.org/corpus/4720.

Santana A. D. (2014). « Virtuous or Vitriolic », Journalism Practice 8, $1: 18-33$.

Silva C. (2011). « Writing in Portuguese chats :): A new wrtng systm? », Written Language \& Literacy $14,1: 143-156$.

Stroebe W., Stroebe M., Abakoumkin G. et Schut H. (1996). « The role of loneliness and social support in adjustment to loss: A test of attachment versus stress theory ", Journal of Personality and Social Psychology 70, $6: 1241-1249$.

Suler J. (2004). « The Online Disinhibition Effect », CyberPsychology \& Behavior 7, 3 : 321-326.

Tanis M. et Postmes T. (2005). « A social identity approach to trust: Interpersonal perception, group membership and trusting behaviour », European Journal of Social Psychology 35 : 413-424.

Taylor L. D. (2010). « Avatars and Emotional Engagement in Asynchronous Online Communication », Cyberpsychology, Behavior, and Social Networking 14, 4 : 207-212.

Temmar M. (2012). « Qui parle dans les blogs? », Manuel d'analyse du web, 126-135.

Tisseron S. (2011). «Intimité et extimité », Communications $88:$ 83-91.

Todorov T. (1981). Mikhail Bakhtine. Le principe dialogique. Suivi de : Ecrits du Cercle de Bakhtine. Paris : Editions du Seuil.

Turkle S. (1984). The Second Self: Computers and the Human Spirit. New York : Simon \& Schuster.

Verheijen L. (2018). Is Textese a Threat to Traditional Literacy? Amsterdam : LOT.

Walther J. B. (1996). « Computer-Mediated Communication: Impersonal, Interpersonal, and Hyperpersonal Interaction », Communication Research 23, 1:3-43.

Walther J. et Boyd S. (2002). « Attraction to computer-mediated social support », Communication Technology and Society: Audience Adoption and Uses, 153-188. 
Wise K., Hamman B. et Thorson K. (2006). « Moderation, Response Rate, and Message

Interactivity: Features of Online Communities and Their Effects on Intent to Participate ", Journal of Computer-Mediated Communication 12, $1: 24-41$.

\section{NOTES}

1. Exemple de liste Topito : http://www.topito.com/top-10-des-trucs-a-ne-pas-oublier-avant-departir-en-vacances.

2. Présentation donnée en 2016 lors d'un séminaire adressé aux animateurs des forums de Doctissimo, les forums dédiées au bien-être et à la santé les plus importants de la francophonie.

\section{RÉSUMÉS}

Cet article définit les spécificités de communication des forums de discussion en replaçant cet environnement au sein d'un tableau de qualification reprenant les 9 principaux environnements de production de contenus numériques que sont les blogs, les forums, les réseaux sociaux, les espaces de commentaires, les sms, les messageries instantanées, les chats, les courriers électroniques et les wikis. Ces environnements sont abordés sur base de 4 critères : le but de la communication, les acteurs, l'(a)synchronie et le pseudonymat. Si l'analyse se centre ici sur les forums, le tableau de qualification peut quant à lui servir de base théorique pour de futures analyses conversationnelles et comparatives des contenus numériques et des nouvelles possibilités d'interaction nées du Web 2.0.

This article aims to define discussion forums specificities of communication by placing this environment in a qualification table including the nine main environments of production of digital contents: blog, forum, social network, wiki, comments section, SMS, instant messaging, chat and email. These environments are analyzed on a basis of 4 criteria: Communication purpose, Participants, Synchronicity and Pseudonymity. This analysis focuses on forums but the qualification table provides a theoretical basis for further conversational analysis of other digital content environments and the enhanced possibilities of interaction raised with Web 2.0.

\section{INDEX}

Keywords : forum, CMC, digital contents, Web 2.0, conversational analysis

Mots-clés : forum, cémo, contenus numériques, web 2.0, analyse conversationnelle

\section{AUTEURS}

ERIKA LOMBART

UCL, ILC, CENTAL, MIIL, FNRS 
CÉDRICK FAIRON

UCL, ILC, CENTAL 